\title{
A microfluidic approach for synthesis and kinetic profiling of branched gold nanostructure
}

Qi Cai ${ }^{a \S}$, Valentina Castagnola ${ }^{a}$, Luca Boselli ${ }^{\S^{*}}$, Alirio Moura $^{b}$, Hender Lopez $^{c}$, Wei Zhang ${ }^{a}$, João M. de Araujo ${ }^{b}$, Kenneth A. Dawson ${ }^{{ }^{*}}$

a. Centre for BioNano Interactions, School of Chemistry, University College Dublin, Belfield, Dublin 4, Ireland.

b. Departamento de Física Teórica e Experimental, Universidade Federal do Rio Grande do Norte, 59078-970, Natal, RN, Brazil.

c. School of Physics and Optometric \& Clinical Sciences, Technological University Dublin, Kevin Street, Dublin 8, Ireland.

\begin{abstract}
Automatized approaches for nanoparticle synthesis and characterization represent a great asset to their applicability in the biomedical field by improving reproducibility and standardization, which help to meet the selection criteria of regulatory authorities. The scaled-up production of nanoparticles with carefully defined characteristics, including intrinsic morphological features, minimal intrabatch, batch-to-batch, and operator variability, is an urgent requirement to elevate nanotechnology towards more trustable biological and technological applications. In this work, microfluidic approaches were employed to achieve fast mixing and good reproducibility in synthesizing a variety of gold nanostructures. The microfluidic setup allowed exploiting spatial resolution to investigate the growth evolution of the complex nanoarchitectures. By physically isolating intermediate reaction fractions, we performed an advanced characterization of the shape properties during their growth, not possible with routine characterization methods. Employing an in-house developed method to assign a specific identity to shapes, we followed the particle growth/deformation process and identified key reaction parameters for more precise control of the generated morphologies. Besides, this investigation led to the optimization of a one-pot multi-size and multi-shape synthesis of a variety of gold nanoparticles. In summary, we describe an optimized platform for highly controlled synthesis and a novel approach for the mechanistic study of shape-evolving nanomaterials.
\end{abstract}

\section{Introduction}

In the last decade, a wide variety of synthetic strategies have been developed to synthesize nanoparticles (NP) with different geometries. ${ }^{1-10}$ Gold nanoparticles (GNP) with complex irregular 
shapes (i.e., branched) present fascinating physicochemical properties, such as a localized surface plasmon resonance peak (LSPR) in the visible-near-infrared (Vis-NIR) spectra and surface-enhanced Raman spectroscopy (SERS) activity, which makes them very attractive for several applications in biology (i.e., imaging, photothermal therapy), sensing and catalysis. ${ }^{11-16}$ Of particular interest is the recently observed shape-dependent biological activity, which could be exploited to design sophisticated tools for interaction with the cellular machinery (including the immune system), promoting an exchange of stimuli-response signals. ${ }^{13-15}$

Several challenges are associated with the production of branched GNP, including the quality of the scaled-up products and the reproducibility (intra-batch and inter-batches shape variability), as an intrinsic consequence of the synthetic process. These factors can profoundly affect the interpretation of biological outcomes/readouts, as well as the clinical and technological transfer of such GNP (including difficulties in patenting specific nanoformulations). ${ }^{17}$ The quality of GNP batches and the final physical features of the particles are strongly affected by the growth route underlying their formation. The study of NP nucleation and growth as complex and stochastic processes has been tackled for many years using complementary approaches. A widely used method to directly capture each step in the nucleation pathway of single particles is in situ transmission electron microscopy (TEM) that allowed following the interactions of the building blocks as they crystallize. ${ }^{18-21}$

Microfluidics approaches for NP synthesis and time-resolved in situ analysis have recently attracted increasing interest. ${ }^{22-30}$ With these methods, the NP synthesis can be carried out in continuous flow using small volumes in an operator-free fashion, and some recent technical advancements, mainly promoted by sensing applications, ${ }^{31-32}$ also allow for in situ microfluidic measurements of physical properties. ${ }^{33-35}$

Here, we developed an in-flow microfluidic synthetic platform to prepare several different highquality GNP with branched shapes. In addition, by exploiting the spatial resolution of the fluidic system to achieve temporal resolution, we investigated reaction pathways for the produced nanoshapes. This novel methodology allowed to precisely isolate reaction intermediates, even at very early times, in a semi-automated fashion and complement the characterization of physicochemical features with morphological analysis. Our approach allowed us to study fast kinetics and provided a complete picture of the intermediate steps that cannot be achieved merely by looking at optical properties. In our recent work, we presented an electron microscopy-based computational method to identify shape signature features and fingerprint the variability of both regular and complex geometrical 3D nano-objects, laying down the first brick for a more ordered understanding of shapedependent interactions at the nanoscale. ${ }^{36-37}$ Here, the shape metrology method is greatly improved, 
allowing for the extraction of multiple geometrical parameters, including size, concavity depth (related to core and tip size), and surface curvature which is proportional to the surface potential. These are important aspects that can allow to control the physicochemical and biological properties of complex nanostructure, as discussed in this work, and potentially impact our future understanding on the surface organization of synthetic functional groups as well as NP-protein interactions and protein corona organization. Furthermore, in the future, this platform might be employed to investigate nanostructure concavities ability to trap small molecules, for example, to build a new generation of highly sensitive biosensors based on plasmon coupling and SERS or to understand key shape parameters to predict and the control of light to heat conversion properties of plasmonic GNPs. Here, by applying the enhanced computational shape analysis method to time-resolved reaction intermediates, we were able to directly analyze/parametrize the evolving structures of the different GNP shapes, building trajectories in the geometrical hyperspace and exploring the growth mechanism, demonstrating how in some cases, this process deviates from a mere size expansion.

Understanding the growth process is essential to produce high-quality NP shapes and move towards controlled engineering of NP morphological properties. This workflow, combining the isolation of the NP at different kinetic stages with the computational method used to classify their shape, and map their surface potential, is intended as a first example to identify some of the critical parameters that can be used to predict the course of the reactions and potentially anticipate the final shape outcomes.

\section{Results}

\section{A novel workflow for synthesis and geometrical characterization of branched gold nanoparticles}

The synthesis of a family of branched GNPs was optimized using different fluidic setups (microreactors - MR, see Figure 1, Supplementary Methods and Figure S1).

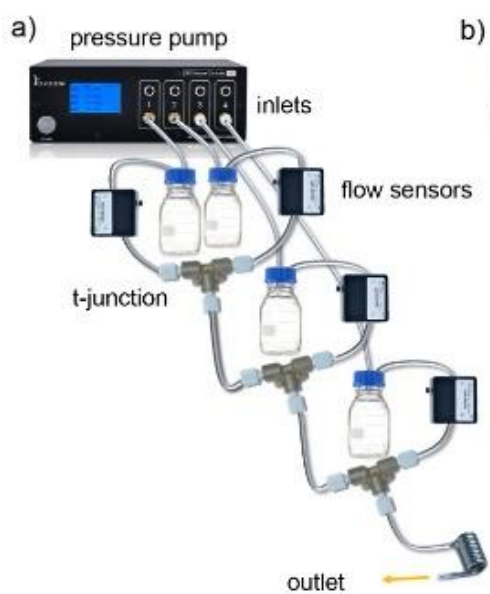

b)

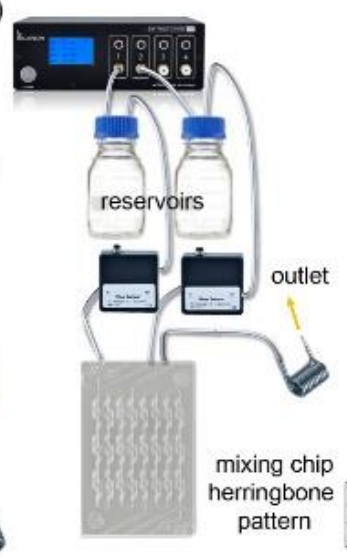

)

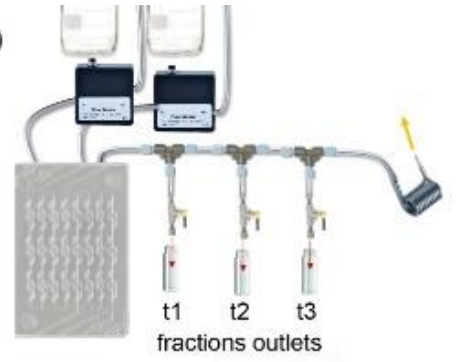

d)

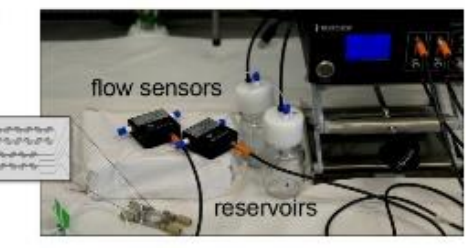


Figure 1. Different microfluidic setups used for the reactions. Schematics of microfluidic setups with, a) four asymmetrical inlet and T-junctions - microfluidic reactor 1 (MR1), b) two symmetrical inlet mixing chip - microfluidic reactor 2 (MR2), c) MR2 used for kinetics studies where the outlets for collection of reaction intermediates are also shown. d) Picture of MR2.

All the synthetic processes were seed-mediated, starting from the same $15 \mathrm{~nm}$ seeds, and were optimized as detailed in Supplementary Methods and Figure S2-5. In Figure 2, the complete characterization of a series of GNP (including branched GNP2-4 and spherical GNP1 control) produced with the microfluidic reactor 1 (MR1) is reported.

GNP1-2_MR1, GNP3_MR1, and GNP4_MR1 were prepared using hydroquinone (HQ), 2,6dihydroxynaphtalene (DHN), and resorcinol (RES) as reducing agents, respectively. The concentration of the reductive hydroxy phenols, the position of their hydroxylate groups (ortho, meta, para), and the number of aromatic rings influence the redox potential and the charge transport capability, affecting the growth mechanism and, therefore, the final shape of the GNP. ${ }^{38}$

Figure 2a depicts a representative TEM micrograph for each GNP produced. In a single TEM image, where the NP contours shape represents the projection of a 3D NP on the grid, dozens of NPs can be analyzed simultaneously. Several different TEM images of the batches (as schematized in Figure 2b) were acquired, and the coordinates of the contours were extracted and analyzed to obtain the shape descriptors (Fourier coefficients obtained by a discrete Fourier transform - DFT - see Experimental Methods). ${ }^{36}$ Considering a NP batch as a collection of nano-objects resulting from a specific synthetic process and containing a variety of nanostructures supposed to have a high similarity among them, we can express the degree of similarity as the "distance" between the shape of each particle and any other particle in the batch (see Experimental and Supplementary Methods for more details). The concept of "distance" between the shape fingerprints (Fourier Spectra) was used to build a cluster analysis based on principal components (PCs) reported in Figure 2c,d. This concept was also used to define shape variance reported in Figure 2e. Finally, the characterization of the optical properties and size distribution was performed using UV-Vis-NIR spectroscopy and differential centrifugal sedimentation (DCS) (Figure 2f,g). 
a)

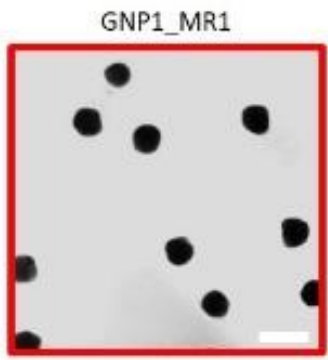

b)

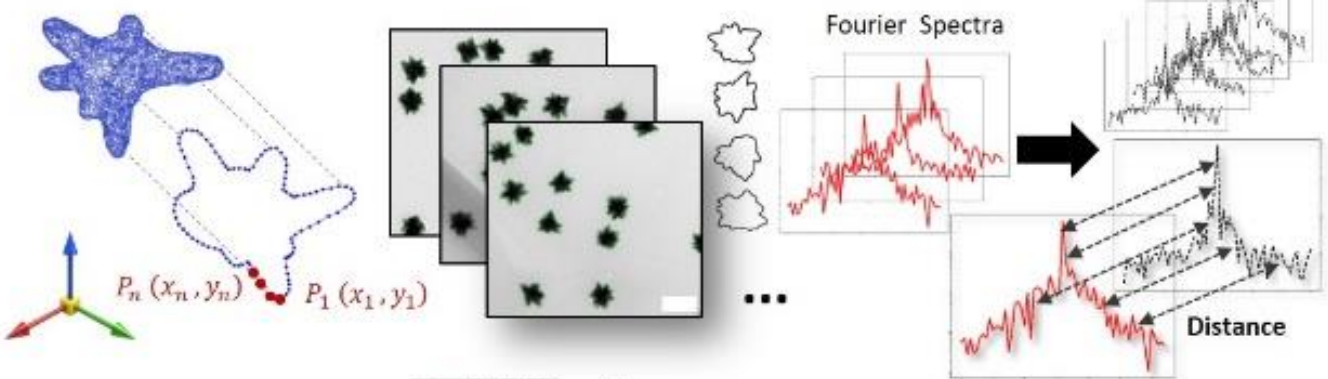

c)

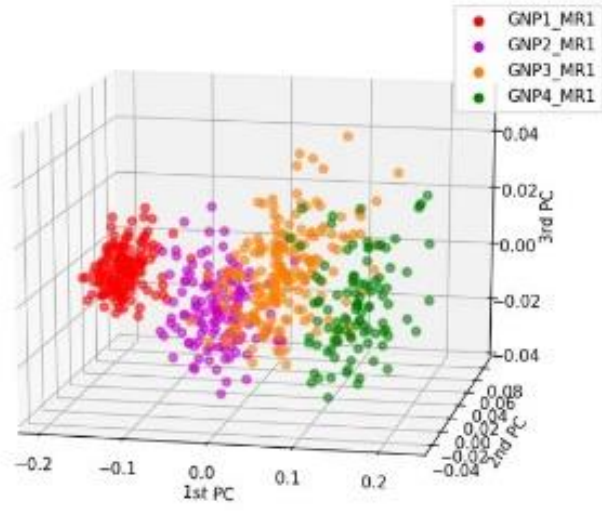

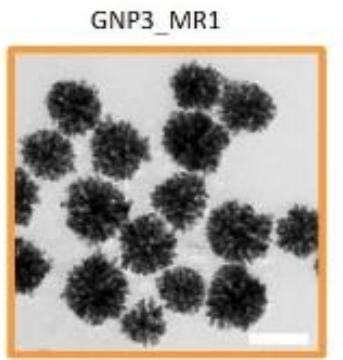

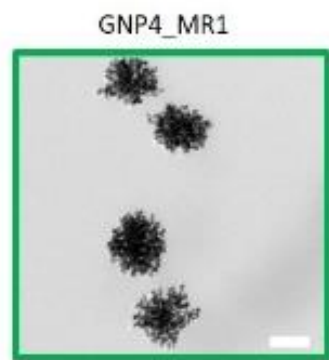

d)

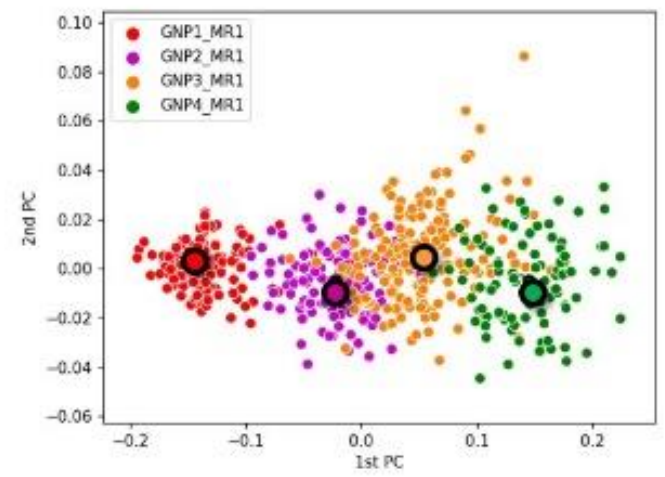

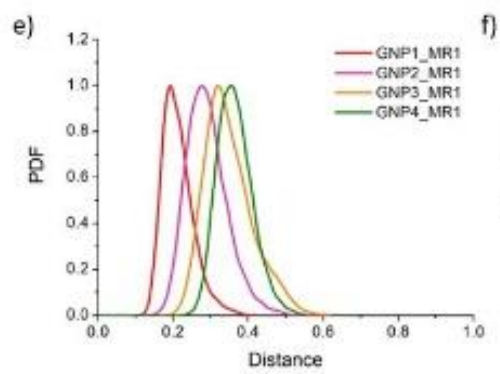
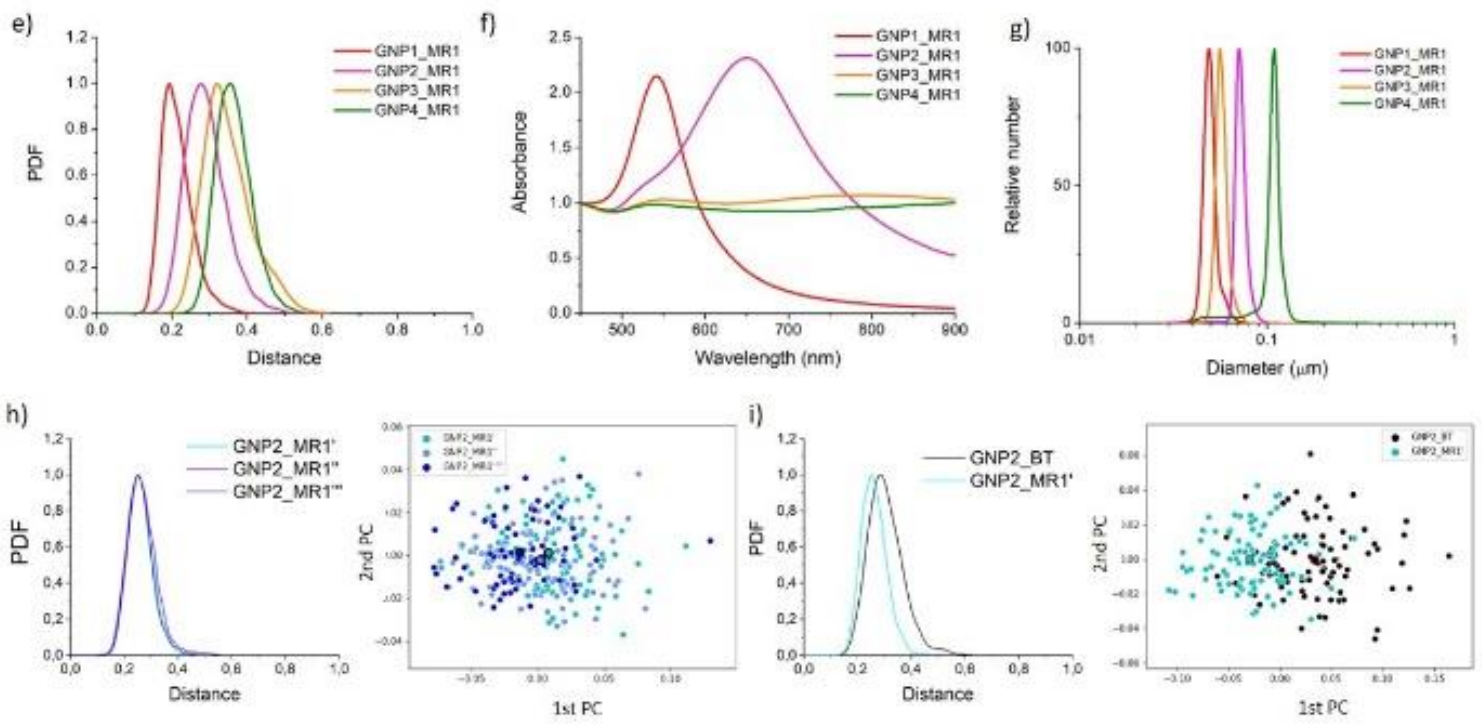

Figure 2. Library of GNP shapes produced using microreactor 1 (MR1). a) Representative TEM micrographs (scale bar is $100 \mathrm{~nm}$ ). b) Schematic of the computational method for shape recognition and assignment: 2D projection of a 3D nanoshape, $X Y$ contours coordinate extraction from TEM imaging, generation of Fourier Spectra, and calculation of the spectra distances. c) 3D and d) 2D scatter plot exhibiting the GNP location in the geometrical 
hyperspace from the principal components (PCs) analysis of the shape. The larger dots with the black border represent the center of gravity of each distribution. e) Shape distributions (shape variance expressed as probability density function -PDF over distance) are obtained by calculating the intra-batch GNP distances. f) UV-Vis-NIR absorption spectra showing LSPR bands for the different shapes normalized at $450 \mathrm{~nm}$. g) DCS analysis showing monomodal GNP apparent size distribution. h) Batch to batch shape reproducibility (GNP2_MR1', GNP2_MR1", GNP2_MR1"') illustrated by PDF plots and scatter plots. i) shape variance improvement from bench-top synthesis (GNP2_BT) and microfluidic synthesis (by MR1) illustrated by PDF plots and scatter plots.

The shape analysis gives us information on the particle shape identity and the intra-batch variability or "shape distribution" (Figure 2c-e). The localization of different clusters in the PC analysis and the shape distribution (or shape variance) as a function of the distances reflect an excellent quality and a precise identity for all the branched GNP produced. In the shape distribution (Figure 2e), the intrabatch particle distances increase with the intrinsic shape complexity. However, all samples exhibited one single population at relatively short intra-particle distances (below 0.5), suggesting that a relatively good shape homogeneity can be achieved despite the irregular geometrical features. As expected, GNP1_MR1, presenting spherical geometry, exhibited sharper shape distribution with low intra-particle distances, while GNP4_MR1 presented the highest shape complexity resulting in more considerable intra-particle distances. Increasing shape complexity/irregularity (i.e., when making multi-branched NP) often leads to an increased shape variance that can generally originate from faster growth rates, which are more "sensitive" to the presence of local inhomogeneities in the reactor (temperature, reagent concentrations, mixing). This also leads to higher batch-to-batch variability. Therefore, the microfluidic approach, by reducing the volume of reactions, and providing an operatorfree continuous flow, clearly limits the local inhomogeneities resulting in more reproducible outcomes. In Figure 2h, an example of shape distribution reproducibility is reported where all the synthesis has been carried out using MR and starting from the same seeds (further examples involving two independent users are reported in Figure S6). The shape variability observed for branched NP is also improved with this method compared to the traditional bench-top approach (Figure 2i).

DCS analysis confirmed monodisperse size distribution for all GNP. The UV-Vis-NIR absorption spectroscopy analysis revealed the characteristic LSPR bands for spherical and branched GNP, the latter typically exhibiting LSPR in the NIR region. ${ }^{39-40}$ However, both GNP3_MR1 and GNP4_MR1 present peculiar LSPR profiles, covering all the Vis-NIR regions without a clear, well-defined peak. 
It is interesting to note that while these two shapes cannot easily be distinguished by visual TEM observation nor absorption spectroscopy, they form two distinct clusters in the shape analysis.

\section{Isolation of reaction intermediates to study the growth kinetics profile}

A simple configuration as MR1 is a fast and cost-effective method to produce high-quality GNP on a large scale (about $150 \mathrm{~mL}$ in $15 \mathrm{~min}$ ). The mixing of the reagents in these conditions, relatively quick due to the small volumes involved, occurs by diffusion (see Supplementary Methods) and probably advection generated by secondary flows due to tubing bending and coiling.

In order to achieve a faster mixing of the reagents (in the milliseconds time scale), and being able to separate the mixing and the reaction area, particularly relevant when aiming to study the kinetics of fast reactions, a micromixer chip was then introduced in the configuration (see MR2 configuration in Supplementary Methods and Figures 1, S1). This modification was essential for studying the early time points, which revealed critical mechanistic features of the reaction.

Following the fast mixing of the gold precursor solution (including the seeds) with the reducing agent solution in the mixing area, the reaction takes place along the outlet tubing in the reaction area, the length of which determines the residence time. The flow rate was optimized to guarantee adequate mixing and proper spatial resolution in the reaction area and achieve good reproducibility (see Figure S6, S7). Using this setup, we followed the growth of four different GNP in-flow reactions, here called DHN1, DHN2, HQ1, HQ2. The nomenclature refers to the type and amount of reducing agent employed (see synthetic details in Supplementary Methods and Table S1).

For a constant flow rate ( 2 or $3 \mathrm{~mL} / \mathrm{min}$, ensured by the flow sensors feedback loop, see Figure S7), we could collect reaction intermediates corresponding to specific time points (see Tables S2) of the reaction by placing flow switches at different outlet tubing lengths. By "freezing" the product of each time point, we could perform a kinetic study with direct in-line isolation of the in-flow evolving sample at different residence times, meaning different stages of the very same starting reaction. To "freeze" the reaction process and isolate the kinetic intermediates, an appropriate capping agent that did not affect the isolated GNP morphologies was selected for each synthesis (see Figures S8-10). All the collected fractions were fully characterized by absorption spectroscopy, DCS, TEM, and shape analysis (see Figure 3 and Figures S11-14).

For reaction DHN1 (Figure 3a-c), it was possible to isolate very early time points, and the spatial resolution allowed for the precise growth pathway reconstruction. The particle evolution revealed a fast kinetic, where after 1 to 3 seconds of reaction, besides a slight growth of the seeds, a rapid formation of dendritic structures decorating the spherical core (seeds) can be observed. The seeds' 
structure appears to support the formation of the branches while maintaining their spherical features, linking the growing extensions through just a few points of contact. The kinetic profile suggests a diffusion-limited reaction, in which fast coalescence of new nuclei formed in the proximity of the NP results in fractal structures. Over time, the branches increase in number and density, leading to a more homogenous final structure and masking the internal spherical core. The TEM images in Figure 3a show complex three-dimensional crystalline hierarchical structures in which the branching sites seem, at least in the first phase, to engage with the seeds almost as individual entities. The complex dendritic structure can be obtained through the oriented attachment of NP over the crystallographic directions (Figure S11a). ${ }^{41}$ The traditional Ostwald ripening mechanism also probably acts to smooth the dendritic morphology over time during the reaction.

We can now have a better interpretation of the peculiar UV-Vis-NIR spectra. The LSPR peak centered at about $540 \mathrm{~nm}$ is continuously present in the spectra over time, presenting a slight red-shift due to the little growth of the core during the first few seconds of reaction. The absorption spectra in the NIR region increase with increasing branches' length and density and, in parallel, with the particles' size. The extended profile of the spectrum, presenting a pretty broad cross-section even over $900 \mathrm{~nm}$, is characteristic of fractal NP conformation in which multiple longitudinal LSPR and intra-particle plasmon coupling occur. ${ }^{42-44}$ By increasing the fractal order, plasmon oscillations related to the different length scales inside the fractal structure increase, including degeneracies. Therefore, fractals can exhibit broad and multi-peaked spectra from plasmons with large degeneracy, which leads to spectrum-spanning.

Both DCS (Figure 3b) and TEM statistical analysis (not reported) show a monomodal size and shape distribution for all the isolated reaction intermediates. Therefore, this strategy can be considered a pioneering approach to preparing multiple GNP batches with different sizes and shapes in a single "one-pot" reaction procedure, avoiding the gold precursor/ reducing agent tuning process commonly performed when aiming to synthesize a series of sizes and shapes. ${ }^{45}$

When using the same configuration, starting from the same reagents but replacing the reducing agent DHN with HQ, the resulting kinetic profile changed utterly. For the reaction called HQ1 (see synthetic details in Experimental Methods), a significant excess of the reducing agent on the gold precursor led to a final product which presents a spiky structure as the one previously observed in GNP2_MR1 and also similar to others obtained by using chemically similar methods (although not inflow). ${ }^{45-46}$ From the kinetic profiling (see the characterization of isolated fractions in Figure S13), it is possible to observe that at early time points (first few seconds), the tips are already clearly visible on the growing seeds, and this morphological change is reflected in the clear red-shift of LSPR compared to 
the one characteristic of spherical GNP of similar sizes. This evidence suggests a direct tip growth over the seeds faces, as confirmed by HR-TEM analysis (see Figure S11b) and other studies reported in the literature. ${ }^{45,47-48}$
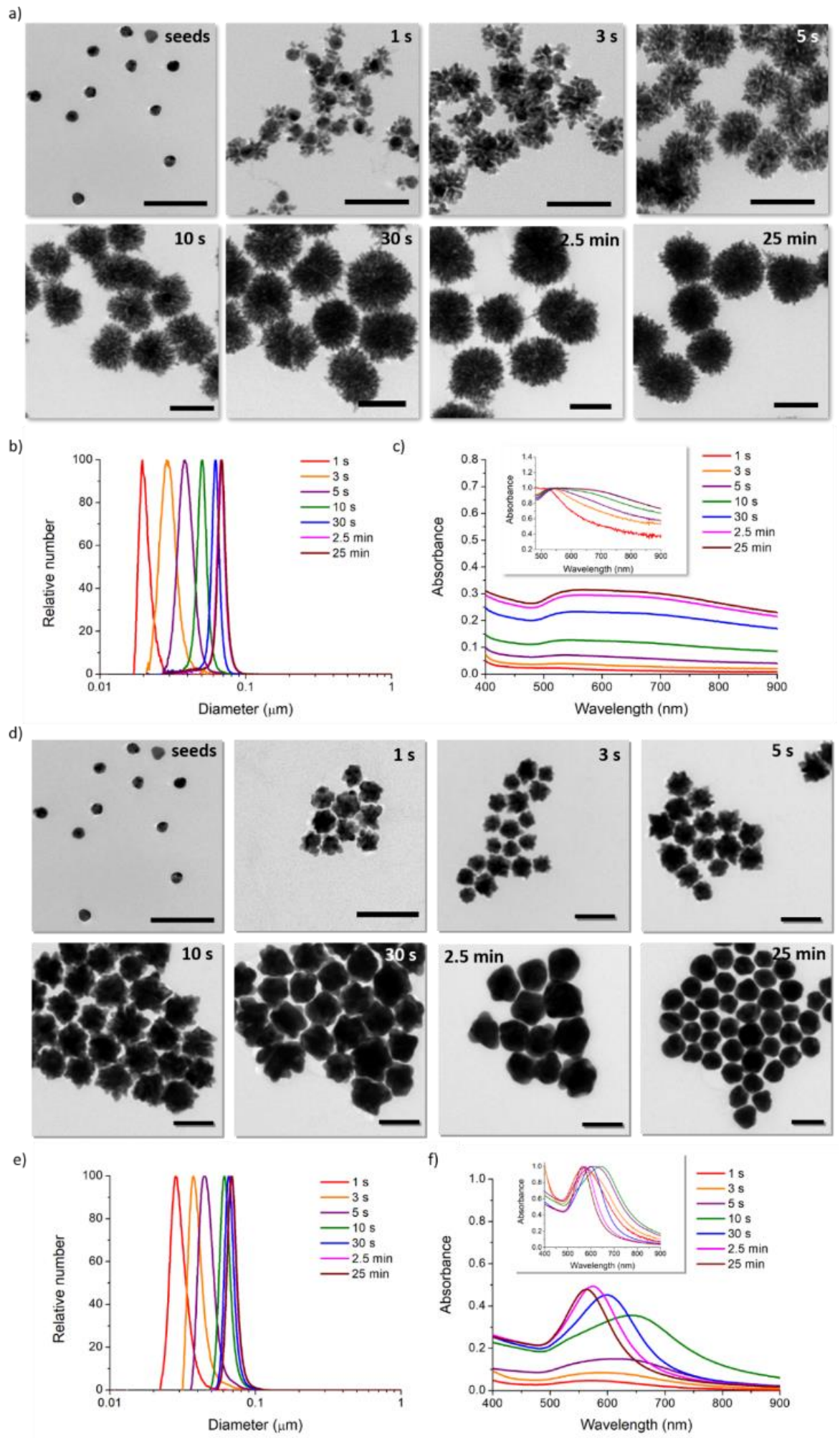

Figure 3. Characterization of the reaction intermediates for microfluidic reaction DHN1 and HQ2 obtained using MR2 setup. For DHN1 reaction intermediates (1 s, 3 s, 5 s, 10 s, 30 s, 2.5 
min, and $25 \mathrm{~min}$ ): a) representative TEM micrographs (scale bars are $100 \mathrm{~nm}$ ); b) DCS analysis; c) UV-Vis-NIR absorption spectra (normalized on LSPR in the inset). For HQ2 reaction intermediates (1 s, $3 \mathrm{~s}, 5 \mathrm{~s}, 10 \mathrm{~s}, 30 \mathrm{~s}, 2.5 \mathrm{~min}$, and $25 \mathrm{~min})$ : d) representative TEM micrographs (scale bars are $100 \mathrm{~nm}$ ); e) DCS analysis; f) UV-Vis-NIR absorption spectra (normalized on LSPR peak in the inset).

A gradual significant overall size growth can be observed from increasing time points, which, however, does not significantly affect the shape defined at a very early growth stage (no further LSPR red-shift is observed after the first 3 seconds of reaction). The mechanism involved appears different from the one observed for DHN1. Here the seeds do not merely act as a template, but they grow in larger structures, meaning that the reduction of the gold is most favored on the surface of the seed and that coalescence can be avoided. This process was previously investigated with traditional approaches, but due to the fast kinetic, a precise analysis of the reaction intermediate (including the LSPR evolution) was not performed. ${ }^{45}$

A more interesting kinetic profile was observed when using a lower concentration of the reducing agents, as in reactions DHN2 (Figure S14) and HQ2 (Figure 3d-f). Both of these synthetic approaches lead to the formation of spherical particles, and in both cases, by capturing the evolution of the shape (TEM imaging) and physical parameters (LSPR), we observed multiple shape transition from spherical seeds to branched/spiky NP and back to a final spherical shape.

\section{Coupling microfluidics synthetic approach and shape analysis platform to study reaction pathways}

As the HQ1 and HQ2 reactions directly involve the growth on the surface and the shape modifications of the seeds, we selected them as suitable models to illustrate the use of our platform for the study of NP growth along the reaction pathway (Figure 4). Some of the chemistry involved in these processes has been previously described elsewhere, ${ }^{49}$ allowing for more robust process reconstruction. Our platform, coupling the microfluidic in-flow isolation of reaction intermediates and the 2D computational shape analysis illustrated before, allows extraction of a series of physical and geometrical parameters in a statistically relevant fashion, which are not accessible using routinely employed characterization techniques (see Supplementary Methods for more details). The intra-batch particle distances and the variance for each reaction time point describe the reaction based on characteristic growth patterns (or reaction fingerprint). In the case of HQ2 (Figure 4a), this pattern 
presents a maximum for distances, while for HQ1 (Figure S15), the reaction moves directly towards the final shape.

As mentioned before, when following the reactions of HQ1 and HQ2, it is possible to isolate a series of GNP batches with increasing size and changing shape (which is a non-trivial task when dealing with non-geometrical nanostructures). As shown in Figure 4 (and Figure S15), this platform allows extracting particle-by-particle statistical parameters directly connected to the GNP branches. In this way, it is relatively easy to follow the evolution of the total size and the branches and, for example, stop the reaction at the desired geometry. In this regard, LSPR is a handy shape-dependent physical parameter strictly related to the branches' development. However, this parameter alone does not allow distinguishing the contribution of size and shape, as similar LSPR maxima can be observed for different shapes (see Figure $2 \mathrm{f}$ and Figure S16). In this case, additional parameters such as the depth and the angle of the inter-tips concavities allow achieving a more detailed picture in the view of linking NP features to their application.

Finally, exploiting the assumption detailed in Supplementary Methods, the surface curvature, which is proportional to the surface chemical potential, can be calculated and plotted for HQ2, as shown in Figure 4d (and Figure S15 for HQ1). This parameter plays a critical role during the growth process of the nanoparticles and, being associated with morphological features, correlates very well with the level of "spikiness" of the shape (here called "concavity factor" and intended as the difference between the surface area of the shape and the circumscriptive convex polygon hull, as shown in Figure S17).

From our experimental observations and previous reports results, it can be hypothesized that in the first stage of the HQ reactions, a large number of gold atoms (with zero-valence) are forming onto the surface of the seeds (nanocrystals), which are acting as surface-catalyzed canters of nucleation (avoiding secondary nucleation in bulk). ${ }^{49-51}$

Here the reaction rate is very high, and most of the gold precursor is consumed during this step. The high concentration of gold atoms instantaneously available allows for preferential growth on specific high-energy facets (presenting larger surface area) of the seed crystals, therefore, leading to anisotropic tips formation. This step occurs far from the equilibrium condition. The early time points of HQ1 and HQ2 (<30 s) indeed clearly show branched nanostructures, but then a different kinetic pathway takes place in the two cases. 
a)

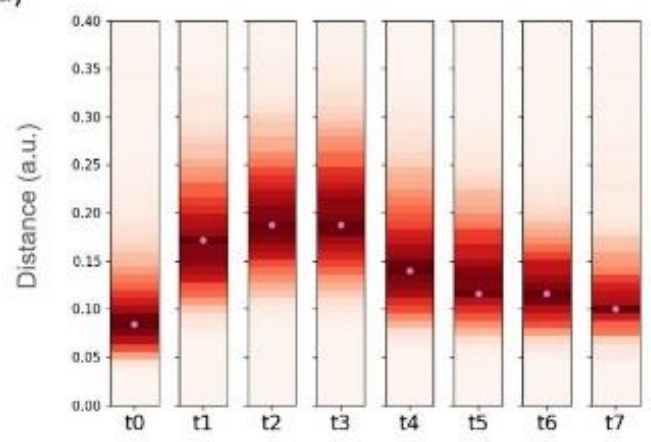

c)

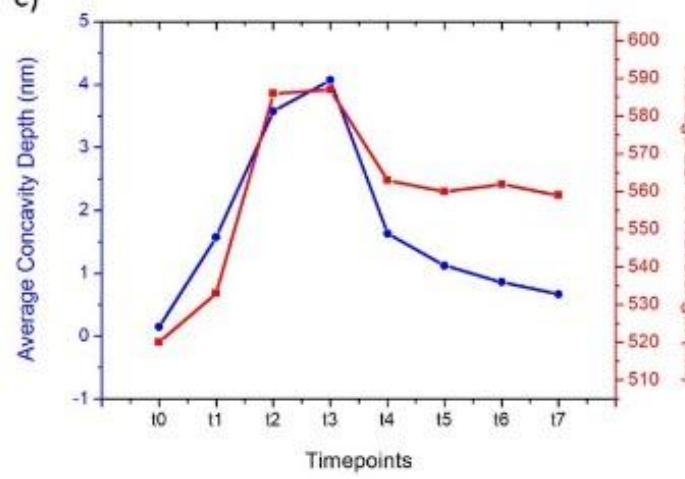

b)

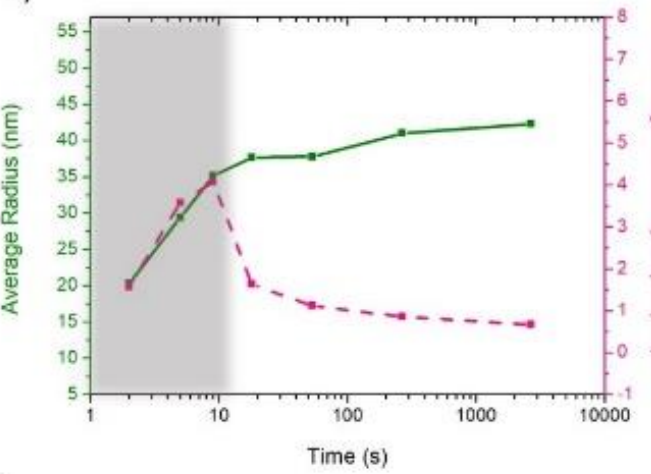

d)

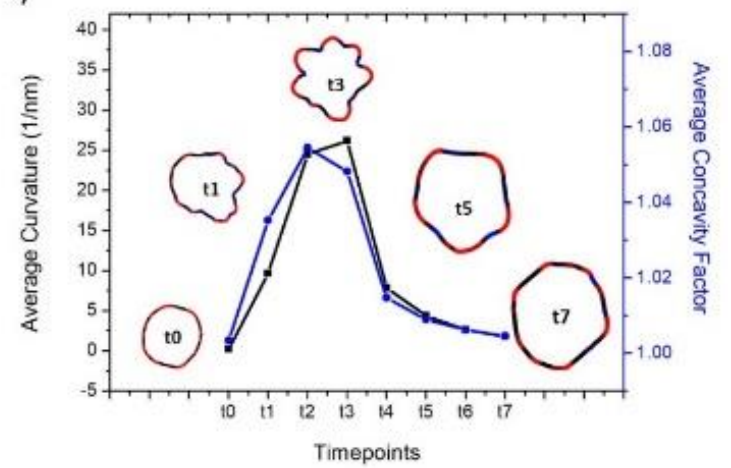

Figure 4. Computational analysis of reaction intermediates for HQ2 synthesis. a) GNP distribution of distances of the Fourier spectra of the GNP contours for each time point (see Table S2 for time points values) for HQ2; b) Average values of radius (green curve) and concavities depth (pink curve) calculated starting from the coordinates of the contour for each time point of HQ2. The trends define two separate regions: first, the total radius and the concavities depth increase together and the GNP shape is defined (gray area), then the radius stabilizes to its final value the concavities are filled (white area). c) Average concavity depth and average LSPR wavelengths for the different time points of HQ2. d) Average surface curvature (proportional to the average surface potential) for each time point of HQ2 calculated as the difference between the curvature of the circumscribed sphere (thermodynamically favorable configuration) and the actual GNP shape. The trend for the surface curvature is compared to the concavity factor. The concavity factor is calculated as the surface area of the GNP over the surface of the convex polygon hull. Representative GNP micrographs for different time points and the related surface curvature (color code: black $=0$, red $=$ positive, blue $=$ negative) are also shown.

Considering this is a diffusion-limited reaction, the decreasing amount of gold concentration over time will also result in a slow-down of the process. Therefore, the gold binding probability decreases 
over time, leading to the expected slower growth at longer time points. For HQ2, there is a crucial turning point in the NP growth in which a critical gold atom concentration (reaction rate) and critical differences in the intra-particle surface potential are reached. From this point, the growth becomes a thermodynamically driven process. In this regime, the most stable structures that try to minimize the total interfacial free energy are produced in a more substantial amount, and we observe a smoothening of the edges, gold filling of concavities, and final spherical shape. The preference of atomic addition to the concave surface can be understood considering the concept of chemical potential, which is defined as the Gibbs free energy per atom. ${ }^{52-54}$ This is comparable to the process described in inert gas or vacuum by the Wulff theorem, where a single crystal assumes the equilibrium shape of the socalled Wulff polyhedron. ${ }^{52,} 55$ Similarly, in our case, the concavity formed during the first stage now presents lower chemical potential than the tips (Figure 4c), and this aspect, combined with the surface mobility (dependent on the capping agent amount and efficacy which is lower for the formed quinone than for starting hydroquinone), plays the principal role in the shape changes and final rearrangement. In contrast, for the case of HQ1, a large excess of HQ reducing/capping agent is employed, and the reaction occurs far from equilibrium until the total consumption of the regent. This process happens with a faster reaction rate and negligible surface atom mobility, which translates to very few rearrangements of the gold atoms, leading to a relatively stable spiky final shape.

Based on these mechanistic hypotheses, it was possible to introduce a mesoscopic kinetic model of the GNP growth based on a deformation algorithm for solids, presented in Figure 5a, Figure S18, and described in detail in Supplementary Methods. This model employs a series of initial conditions from experimental data (such as gold precursor/seeds ratio, reducing agent/capping agent amount), reasonable assumptions (such as the increasing probability that reduced gold attaches on larger facets) and one free parameter here defined as "surface rearrangement", which is influenced by multiple factors (such as gold atoms mobility, surface stress, and strain, capping agent, temperature, chemical environment). ${ }^{52,56-61}$ 2D particle projections were extracted from the in silico grown $3 \mathrm{D}$ structures (see Figure 5a) for each time point along with the reaction coordinates and compared with the experimental results (Figure $5 \mathrm{~b}$ and Figure S19). The free parameter of the model (the surface rearrangement) is tuned to obtain the final shape of each reaction (HQ1 or HQ2), providing a good match between the extrapolated and the experimental time points.

From the model, it is possible to extract advanced statistical information such as the volume growth and the reaction rate (Figure 5c and Figure S19), avoiding extremely time-consuming processes such as the 3D-tomography reconstruction of hundreds of particles which, given the complexity of the structures would lead to approximate results. 
a)

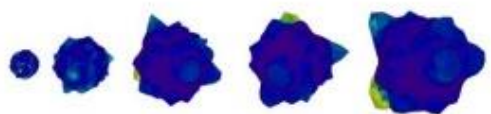

b)

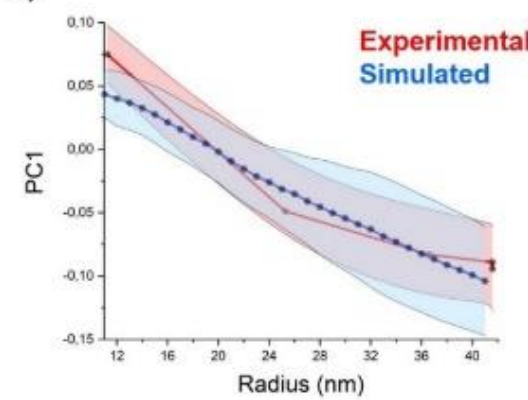

c)

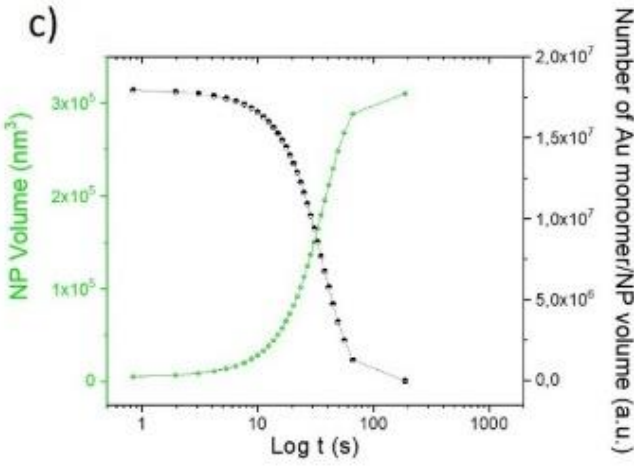

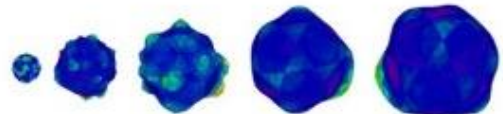
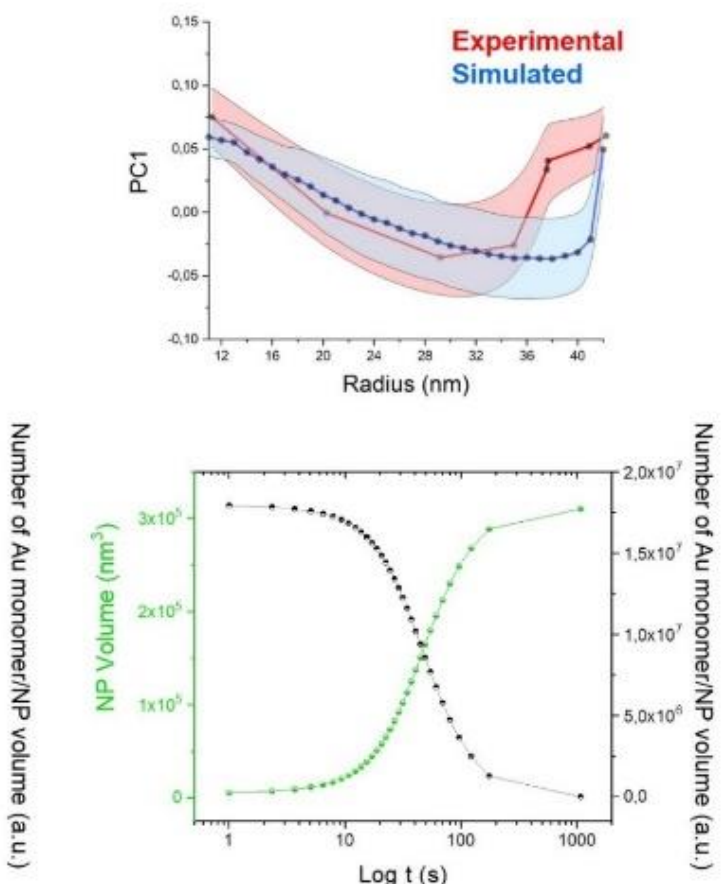

Figure 5. Mesoscopic model of the growth kinetic for HQ1 (left) and HQ2 (right). a) 3D particle reconstruction for increasing time points, b) first principal component for the experimental time points (red curve), and ten simulations starting from different seeds (blue curves). The ribbons represent the standard deviations. c) Volume growth rate (green) and gold consumption (black) over time.

Therefore, our model might help predict some intermediate growth shapes and could be used as an exploratory tool to assist the synthesis and design on new nanostructure and we believe in the future could help to predict optical, catalytic, and with a series of reiterative processes even biological properties (i.e., adjuvancy).

\section{Conclusion}

With the present work, we introduced a novel in-flow approach to isolate and characterize reaction intermediated from continuous flow synthesis of inorganic NP with complex geometries. This approach provides the unique chance to describe the NP growth while happening, producing a variety of branched GNP (some have never been reported before or never been prepared using this method) that are presenting distinguishable features. Exploiting the spatial resolution allowed by the fluidic 
reactor and the fast mixing, it was possible to capture multiple intermediates of reaction for a given synthetic process, including early time points. This methodology also allowed to synthesize GNP batches with different sizes and shapes simultaneously in a single-step "one-pot" reaction. The GNP and their reaction intermediates were analyzed by a recently developed method based on extrapolation of key physical and geometrical features (size, branches geometry, surface potential) from the 2D projection of the particles obtained by TEM imaging. The data obtained by this treatment allow for more precise identification of nanoshapes and reaction pathways but also helped to develop a 3D model of the NP growth process. The proposed mesoscopic model of the growth kinetic can be used to predict the final shape of the NP depending on the time of the reaction, which can potentially be used to isolate selected shapes intermediates during the reaction and predict products for unexplored chemical combinations, but further improvements are needed to achieve for more precise predictions. In this regard, the microfluidic approach opens up to intriguing opportunities to monitor and isolate specific morphologies. With the combination of microfluidics and computational tools here reported, we aim to pave the way for a novel paradigm of shape generation, including enhanced control over the quality, reproducibility, and final geometry, still lacking nowadays for most of the synthetic approaches. Precise control of the produced nanoshapes is especially relevant in order to correlate outcomes in biology, sensing, and catalysis with intrinsic NP morphological features and achieve a precise tuning of such features based on the targeted application.

\section{Experimental section}

\section{Synthesis of GNP1-4 using MR1}

Four glass bottle reservoirs containing $50 \mathrm{~mL}$ of reagents were used. All the glassware and stir bars were previously cleaned with aqua regia and thoroughly rinsed with fresh Milli-Q water.

The reaction took place in PTFE tubing and T-junctions. Four flow sensors were placed between reservoirs and T-junctions to monitor the flow rate. The tubing length between the reservoir and the flow sensor was $30 \mathrm{~cm}$. The flow sensors were connected to a T-junction with $10 \mathrm{~cm}$ tubing while the outlet tubing was $15 \mathrm{~m}$ long. The total flow rate used in the synthesis was $10 \mathrm{~mL} / \mathrm{min}$, and the ratio of the flow rate of the four inlets is $1: 1: 1: 1$.

All the syntheses were seed-mediated, and the $15 \mathrm{~nm}$ seeds described above were used. The amount of $\mathrm{HAuCl}_{4}$ and trisodium citrate $\left(\mathrm{Na}_{3} \mathrm{Ct}\right)$ was kept constant for all the GNP, specifically:

- Reservoir 1: $50 \mathrm{~mL}$ of an aqueous solution of $\mathrm{HAuCl}_{4}\left(5 \times 10^{-5} \mathrm{~mol}, 1 \times 10^{-3} \mathrm{M}\right)$;

- Reservoir 3: $50 \mathrm{~mL}$ of an aqueous solution of $\mathrm{Na}_{3} \mathrm{Ct}\left(1.5 \times 10^{-5} \mathrm{~mol}, 3 \times 10^{-4} \mathrm{M}\right)$;

The amount of reducing agent and seeds was varied, as detailed below.

\section{GNP1_MR1}

- Reservoir 2: $8 \mathrm{~mL}$ of seeds from the stock $\left(8 \times 10^{-12} \mathrm{~mol}\right)$ was added to $42 \mathrm{~mL}$ of $\mathrm{H}_{2} \mathrm{O}$ for a final concentration of $1.6 \times 10^{-10} \mathrm{M}$;

- Reservoir 4: $50 \mathrm{~mL}$ of an aqueous solution of HQ $\left(6 \times 10^{-5} \mathrm{~mol}, 1.2 \times 10^{-3} \mathrm{M}\right)$. 


\section{GNP2_MR1}

- Reservoir 2: $1 \mathrm{~mL}$ of seeds from the stock $\left(1 \times 10^{-12} \mathrm{~mol}\right)$ was added to $49 \mathrm{~mL}$ of $\mathrm{H}_{2} \mathrm{O}$ for a final concentration of $2 \times 10^{-11} \mathrm{M}$;

- Reservoir 4: $50 \mathrm{~mL}$ of an aqueous solution of HQ $\left(6 \times 10^{-4} \mathrm{~mol}, 1.2 \times 10^{-2} \mathrm{M}\right)$.

\section{GNP3_MR1}

- Reservoir 2: $3 \mathrm{~mL}$ of seeds from the stock $\left(3 \times 10^{-12}\right.$ mol) was added to $47 \mathrm{~mL}$ of $\mathrm{H}_{2} \mathrm{O}$ for a final concentration of $6 \times 10^{-11} \mathrm{M}$;

- Reservoir 4: $50 \mathrm{~mL}$ of an aqueous solution of DHN $\left(6 \times 10^{-4} \mathrm{~mol}, 1.2 \times 10^{-2} \mathrm{M}\right)$.

\section{GNP4_MR1}

For the synthesis of GNP4_MR1, the outlet tube was placed in a $40^{\circ} \mathrm{C}$ water bath. The temperature was stabilized for $20 \mathrm{~min}$ before the experiment.

- Reservoir 2: $2 \mathrm{~mL}$ of seeds from the stock $\left(2 \times 10^{-12} \mathrm{~mol}\right)$ was added to $48 \mathrm{~mL}$ of $\mathrm{H}_{2} \mathrm{O}$ for a final concentration of $4 \times 10^{-11} \mathrm{M}$;

- Reservoir 4: $50 \mathrm{~mL}$ of aqueous RES solution $\left(6 \times 10^{-4} \mathrm{~mol}, 1.2 \times 10^{-2} \mathrm{M}\right)$.

\section{Synthesis of HQ1, HQ2, DHN1, and DHN2}

The NPs were synthesized using MR2 via seed-mediated growth methods starting from the $15 \mathrm{~nm}$ gold seeds, as described before. All the glassware and stir bars were previously cleaned with aqua regia and thoroughly rinsed with fresh Milli-Q water. The concentrations of the reagent solutions used are summarized in Table S1.

Milli-Q water used was degassed under vacuum for $30 \mathrm{~min}$ and stored at $4{ }^{\circ} \mathrm{C}$ prior to use for all reagent solutions. Glycerol was added into water (about $250 \mu \mathrm{L}$ of glycerol in $100 \mathrm{~mL}$ of water) to prevent the formation of ice crystals during the experiment. Two glass bottle reservoirs with a total volume of $20 \mathrm{~mL}$ each were placed in an ice bath to slow down possible reactions inside the reservoirs. A micromixer chip was used as described before and showed in Figures S1-2. Two flow sensors were placed between the reservoirs and the mixing chip. The mixing chip was placed on the TCU set to $1{ }^{\circ} \mathrm{C}$ and left to stabilize for $15 \mathrm{~min}$ before starting the experiment. The tubing length between the reservoir and the flow sensor was $30 \mathrm{~cm}$, and it was $10 \mathrm{~cm}$ between the flow sensors and the mixing chip. The flow rate ratio between inlet 1 and inlet 2 was $1: 1$, and the total flow rate was 3 $\mathrm{mL} / \mathrm{min}$ for HQ1, HQ2, and DHN1, and it was $2 \mathrm{~mL} / \mathrm{min}$ for DHN2.

\section{Reaction intermediates isolation}

Luer-lock T-junction and microfluidic sample injection shut-off valves were placed at established distances on the outlet tube (as shown in Figure S1.c). The distances between the valves and the starting point of the outlet tube determined different time points based on the actual flow rate recorded. The flow rate ratio between inlet 1 and inlet 2 was 1:1, and the total flow rate was $3 \mathrm{~mL} / \mathrm{min}$ for HQ1, HQ2, and DHN1, and $2 \mathrm{~mL} / \mathrm{min}$ for DHN2. About $1 \mathrm{~mL}$ for each fraction was collected in $14 \mathrm{~mL}$ glass vials containing $200 \mu \mathrm{L}$ of thiol ligand solution $\left(2 \times 10^{-5} \mathrm{~mol}, 0.01 \mathrm{M}\right.$, PEG ligand for 
HQ1 and HQ2; $2 \times 10^{-5} \mathrm{~mol}, 0.01 \mathrm{M}$ of 3-MPA ligand for DHN1 and DHN2). An aliquot of the final fraction was left without the thiol ligand solution for $15 \mathrm{~min}$ to verify reaction completion.

After collection, all the fractions were diluted in Milli-Q water and washed multiple times by centrifugal filters (Sartorius, $10 \mathrm{KDa}$ ) to remove unreacted reagents.

\section{Nanoparticles contour extraction from TEM imaging}

According to a protocol described elsewhere,${ }^{36}$ most of the samples used for the shape analysis were deposited onto the TEM grids, which allows for nanoparticles spreading and avoids aggregation due to drying effects. Briefly, the samples were diluted to the concentration of $1-3 \times 10^{11} \mathrm{NP} / \mathrm{mL}$ and incubated at R.T. in $0.2 \%$ methylcellulose overnight. Ultrathin carbon on holey 400 mesh $\mathrm{Cu}$ grids (Ted Pella Inc.) was previously treated with a glow discharger were used. Images were taken on a FEI Tecnai G2 20 Twin operating at $120 \mathrm{kV}$, at magnifications between 29000x and 62000x.

For few TEM images deposited by drop-casting without methylcellulose pre-incubation, some pretreatment was necessary to extract the contours. Therefore, some TEM micrographs were slightly modified using the free software GIMP 2.10.12.

\section{Shape analysis of the 2D NP contours}

TEM micrographs were used to analyze the shape of different batches of GNP, following a procedure described elsewhere. ${ }^{36}$ Briefly, the GNP contours were obtained from the TEM images using the Open Source Computer Vision Library (OpenCV). The extracted contours consist of closed 2D curves, which coordinates define the GNP contours. The coordinates were then used to perform a Discrete Fourier Transform (DFT) of each contour. In order to define shape clusters, we employed either the Fourier coefficients (FCs) or the Principal Components (PC) extracted from a principal component analysis (PCA) of the FCs. The probability distribution function (PDF) of the shapes was obtained from the $L_{1}$ norm distances between all the FCs in a batch. For further details on the contour extraction and the shape analysis, see our previous publication. ${ }^{36}$

\section{Acknowledgments}

H. L., V.C., and Q.C. acknowledge financial support from the Irish Research Council (H.L., EPSPD/2015/5; V.C., GOIPD/2016/128; Q.C., GOIPG/2014/874). Q.C. and W.Z acknowledge the Chinese Scholarship Council (agreement no. 201408300003 and 201706220062). J.M.A. acknowledges CNPq for support through Bolsa de Produtividade em Pesquisa No. 313431/2018- 3. Access to and use of the UCD Conway Electron Microscopy Facility is gratefully acknowledged. The authors acknowledge Dr. Sean O’Brien and Dr. Camila Pedroso Silveira. 


\section{References}

1. Grzelczak, M.; Pérez-Juste, J.; Mulvaney, P.; Liz-Marzán, L. M., Shape control in gold nanoparticle synthesis. Chemical Society Reviews 2008, 37 (9), 1783-1791.

2. Hao, E.; Schatz, G. C.; Hupp, J. T., Synthesis and optical properties of anisotropic metal nanoparticles. Journal of Fluorescence 2004, 14 (4), 331-341.

3. Sau, T. K.; Murphy, C. J., Room temperature, high-yield synthesis of multiple shapes of gold nanoparticles in aqueous solution. Journal of the American Chemical Society 2004, 126 (28), 8648-8649.

4. Yuan, H.; Khoury, C. G.; Hwang, H.; Wilson, C. M.; Grant, G. A.; Vo-Dinh, T., Gold nanostars: surfactant-free synthesis, 3D modelling, and two-photon photoluminescence imaging. Nanotechnology 2012, 23 (7), 075102.

5. Ye, X.; Jin, L.; Caglayan, H.; Chen, J.; Xing, G.; Zheng, C.; Doan-Nguyen, V.; Kang, Y.; Engheta, N.; Kagan, C. R., Improved size-tunable synthesis of monodisperse gold nanorods through the use of aromatic additives. ACS nano 2012, 6 (3), 2804-2817.

6. Manna, L.; Scher, E. C.; Alivisatos, A. P., Synthesis of soluble and processable rod-, arrow-, teardrop, and tetrapod-shaped CdSe nanocrystals. Journal of the American Chemical Society 2000, 122 (51), 1270012706.

7. Sun, Y.; Xia, Y., Shape-controlled synthesis of gold and silver nanoparticles. Science 2002, 298 (5601), 2176-2179.

8. Ahmadi, T. S.; Wang, Z. L.; Green, T. C.; Henglein, A.; El-Sayed, M. A., Shape-controlled synthesis of colloidal platinum nanoparticles. Science 1996, 272 (5270), 1924.

9. Jana, N. R.; Gearheart, L.; Murphy, C. J., Seed-mediated growth approach for shape-controlled synthesis of spheroidal and rod-like gold nanoparticles using a surfactant template. Advanced Materials 2001, 13 (18), 1389.

10. Maiorano, G.; Rizzello, L.; Malvindi, M. A.; Shankar, S. S.; Martiradonna, L.; Falqui, A.; Cingolani, R.; Pompa, P. P., Monodispersed and size-controlled multibranched gold nanoparticles with nanoscale tuning of surface morphology. Nanoscale 2011, 3 (5), 2227-2232.

11. Jeong, G. H.; Lee, Y. W.; Kim, M.; Han, S. W., High-yield synthesis of multi-branched gold nanoparticles and their surface-enhanced Raman scattering properties. Journal of colloid and interface science 2009, $329(1), 97-102$.

12. Xie, J.; Zhang, Q.; Lee, J. Y.; Wang, D. I., The synthesis of SERS-active gold nanoflower tags for in vivo applications. ACS nano 2008, 2 (12), 2473-2480.

13. Talamini, L.; Violatto, M. B.; Cai, Q.; Monopoli, M. P.; Kantner, K.; Krpetic, Z.; Perez-Potti, A.; Cookman, J.; Garry, D.; P. Silveira, C., Influence of size and shape on the anatomical distribution of endotoxin-free gold nanoparticles. ACS nano 2017, 5519-5529.

14. Albanese, A.; Sykes, E. A.; Chan, W. C., Rough around the edges: the inflammatory response of microglial cells to spiky nanoparticles. ACS nano 2010, 4 (5), 2490-2493.

15. Wang, J.; Chen, H. J.; Hang, T.; Yu, Y.; Liu, G.; He, G.; Xiao, S.; Yang, B. R.; Yang, C.; Liu, F.; Tao, J.; Wu, M. X.; Xie, X., Physical activation of innate immunity by spiky particles. Nat Nanotechnol 2018, 13 (11), 1078-1086.

16. Donati, P.; Pomili, T.; Boselli, L.; Pompa, P. P., Colorimetric Nanoplasmonics to Spot Hyperglycemia From Saliva. Frontiers in bioengineering and biotechnology 2020, 8, 1404.

17. Faria, M.; Björnmalm, M.; Thurecht, K. J.; Kent, S. J.; Parton, R. G.; Kavallaris, M.; Johnston, A. P.; Gooding, J. J.; Corrie, S. R.; Boyd, B. J., Minimum information reporting in bio-nano experimental literature. Nature nanotechnology 2018, 13 (9), 777-785.

18. Tang, M. L.; Liu, N.; Dionne, J. A.; Alivisatos, A. P., Observations of shape-dependent hydrogen uptake trajectories from single nanocrystals. Journal of the American Chemical Society 2011, 133 (34), 13220-13223.

19. Zheng, H.; Smith, R. K.; Jun, Y.-w.; Kisielowski, C.; Dahmen, U.; Alivisatos, A. P., Observation of single colloidal platinum nanocrystal growth trajectories. science 2009, 324 (5932), 1309-1312.

20. Woehl, T. J.; Evans, J. E.; Arslan, I.; Ristenpart, W. D.; Browning, N. D., Direct in situ determination of the mechanisms controlling nanoparticle nucleation and growth. ACS nano 2012, 6 (10), 8599-8610. 
21. Ou, Z.; Wang, Z.; Luo, B.; Luijten, E.; Chen, Q., Kinetic pathways of crystallization at the nanoscale. Nature materials 2020, 19 (4), 450-455.

22. Edel, J. B.; Fortt, R., Microfluidic routes to the controlled production of nanoparticles. Chemical Communications 2002, (10), 1136-1137.

23. Chan, E. M.; Mathies, R. A.; Alivisatos, A. P., Size-controlled growth of CdSe nanocrystals in microfluidic reactors. Nano Letters 2003, 3 (2), 199-201.

24. Huang, H.; du Toit, H.; Ben-Jaber, S.; Wu, G. W.; Panariello, L.; Thanh, N. T. K.; Parkin, I. P.; Gavriilidis, A., Rapid synthesis of gold nanoparticles with carbon monoxide in a microfluidic segmented flow system. Reaction Chemistry \& Engineering 2019, 4 (5), 884-890.

25. Abalde-Cela, S.; Taladriz-Blanco, P.; de Oliveira, M. G.; Abell, C., Droplet microfluidics for the highly controlled synthesis of branched gold nanoparticles. Scientific reports 2018, 8 (1), 2440.

26. Abdel-Latif, K.; Epps, R. W.; Kerr, C. B.; Papa, C. M.; Castellano, F. N.; Abolhasani, M., Facile RoomTemperature Anion Exchange Reactions of Inorganic Perovskite Quantum Dots Enabled by a Modular Microfluidic Platform. Advanced Functional Materials 2019, 1900712.

27. Alloyeau, D.; Dachraoui, W.; Javed, Y.; Belkahla, H.; Wang, G.; Lecoq, H. I. n.; Ammar, S.; Ersen, O.; Wisnet, A.; Gazeau, F., Unravelling kinetic and thermodynamic effects on the growth of gold nanoplates by liquid transmission electron microscopy. Nano letters 2015, 15 (4), 2574-2581.

28. Rubio-Martinez, M.; Imaz, I.; Domingo, N.; Abrishamkar, A.; Mayor, T. S.; Rossi, R. M.; Carbonell, C.; deMello, A. J.; Amabilino, D. B.; Maspoch, D., Freezing the nonclassical crystal growth of a coordination polymer using controlled dynamic gradients. Advanced Materials 2016, 28 (37), 8150-8155.

29. Sevim, S.; Sorrenti, A.; Franco, C.; Furukawa, S.; Pané, S.; Demello, A.; Puigmartí-Luis, J., Selfassembled materials and supramolecular chemistry within microfluidic environments: from common thermodynamic states to non-equilibrium structures. Chemical Society Reviews 2018, 47 (11), 3788-3803.

30. Nette, J.; Howes, P. D.; deMello, A. J., Microfluidic Synthesis of Luminescent and Plasmonic Nanoparticles: Fast, Efficient, and Data-Rich. Advanced Materials Technologies 2020, 5 (7), 2000060.

31. Parisi, J.; Dong, Q.; Lei, Y., In situ microfluidic fabrication of SERS nanostructures for highly sensitive fingerprint microfluidic-SERS sensing. RSC advances 2015, 5 (19), 14081-14089.

32. Parisi, J.; Su, L.; Lei, Y., In situ synthesis of silver nanoparticle decorated vertical nanowalls in a microfluidic device for ultrasensitive in-channel SERS sensing. Lab on a Chip 2013, 13 (8), 1501-1508.

33. Seibt, S.; Mulvaney, P.; Förster, S., Millisecond CdS nanocrystal nucleation and growth studied by microfluidics with in situ spectroscopy. Colloids and Surfaces A: Physicochemical and Engineering Aspects 2019, 562, 263-269.

34. Herbst, M.; Hofmann, E.; Förster, S., Nucleation and Growth Kinetics of ZnO Nanoparticles Studied by in Situ Microfluidic SAXS/WAXS/UV-Vis Experiments. Langmuir 2019, 35 (36), 11702-11709.

35. Merkens, S.; Vakili, M.; Sánchez-Iglesias, A.; Litti, L.; Gao, Y.; Gwozdz, P. V.; Sharpnack, L.; Blick, R. H.; Liz-Marzán, L. M.; Grzelczak, M., Time-Resolved Analysis of the Structural Dynamics of Assembling Gold Nanoparticles. ACS Nano 2019, 13 (6), 6596-6604.

36. Boselli, L.; Lopez, H.; Zhang, W.; Cai, Q.; Giannone, V. A.; Li, J.; Moura, A.; de Araujo, J. M.; Cookman, J.; Castagnola, V., Classification and biological identity of complex nano shapes. Communications Materials 2020, 1 (1), 1-12.

37. Calvaresi, M., The route towards nanoparticle shape metrology. Nature nanotechnology 2020, 15 (7), 512-513.

38. Lee, Y.; Park, T. G., Facile fabrication of branched gold nanoparticles by reductive hydroxyphenol derivatives. Langmuir 2011, 27 (6), 2965-2971.

39. Trigari, S.; Rindi, A.; Margheri, G.; Sottini, S.; Dellepiane, G.; Giorgetti, E., Synthesis and modelling of gold nanostars with tunable morphology and extinction spectrum. Journal of Materials Chemistry 2011, 21 (18), 6531-6540.

40. Kelly, K. L.; Coronado, E.; Zhao, L. L.; Schatz, G. C., The optical properties of metal nanoparticles: the influence of size, shape, and dielectric environment. The Journal of Physical Chemistry B 2003, 107 (3), 668677.

41. Cheng, Y.; Wang, Y.; Chen, D.; Bao, F., Evolution of single crystalline dendrites from nanoparticles through oriented attachment. The Journal of Physical Chemistry B 2005, 109 (2), 794-798. 
42. Kim, J.; Liu, G. L.; Lu, Y.; Lee, L. P., Intra-particle plasmonic coupling of tip and cavity resonance modes in metallic apertured nanocavities. Optics express 2005, 13 (21), 8332-8338.

43. Gottheim, S.; Zhang, H.; Govorov, A. O.; Halas, N. J., Fractal nanoparticle plasmonics: The Cayley tree. ACS nano 2015, 9 (3), 3284-3292.

44. Kim, S.; Shuford, K. L.; Bok, H.-M.; Kim, S. K.; Park, S., Intraparticle surface plasmon coupling in quasi-one-dimensional nanostructures. Nano letters 2008, 8 (3), 800-804.

45. Li, J.; Wu, J.; Zhang, X.; Liu, Y.; Zhou, D.; Sun, H.; Zhang, H.; Yang, B., Controllable synthesis of stable urchin-like gold nanoparticles using hydroquinone to tune the reactivity of gold chloride. The Journal of Physical Chemistry C 2011, 115 (9), 3630-3637.

46. Potenza, M.; Krpetić, Ž.; Sanvito, T.; Cai, Q.; Monopoli, M.; de Araújo, J.; Cella, C.; Boselli, L.; Castagnola, V.; Milani, P., Detecting the shape of anisotropic gold nanoparticles in dispersion with single particle extinction and scattering. Nanoscale 2017, 9 (8), 2778-2784.

47. Lv, W.; Gu, C.; Zeng, S.; Han, J.; Jiang, T.; Zhou, J., One-Pot Synthesis of Multi-Branch Gold Nanoparticles and Investigation of Their SERS Performance. Biosensors 2018, 8 (4), 113.

48. Saverot, S.; Geng, X.; Leng, W.; Vikesland, P.; Grove, T.; Bickford, L., Facile, tunable, and SERSenhanced HEPES gold nanostars. RSC Advances 2016, 6 (35), 29669-29673.

49. Perrault, S. D.; Chan, W. C., Synthesis and surface modification of highly monodispersed, spherical gold nanoparticles of 50- $200 \mathrm{~nm}$. Journal of the American Chemical Society 2009, 131 (47), 17042-17043.

50. Mostafavi, M.; Marignier, J.; Amblard, J.; Belloni, J., Nucleation dynamics of silver aggregates simulation of photographic development processes. International Journal of Radiation Applications and Instrumentation. Part C. Radiation Physics and Chemistry 1989, 34 (4), 605-617.

51. Linnert, T.; Mulvaney, P.; Henglein, A.; Weller, H., Long-lived nonmetallic silver clusters in aqueous solution: preparation and photolysis. Journal of the American Chemical Society 1990, 112 (12), 4657-4664.

52. Xia, Y.; Xiong, Y.; Lim, B.; Skrabalak, S. E., Shape-controlled synthesis of metal nanocrystals: simple chemistry meets complex physics? Angewandte Chemie International Edition 2009, 48 (1), 60-103.

53. Keys, A. S.; lacovella, C. R.; Glotzer, S. C., Characterizing complex particle morphologies through shape matching: Descriptors, applications, and algorithms. Journal of Computational Physics 2011, 230 (17), 6438-6463.

54. Ouyang, G.; Wang, C.; Yang, G., Surface energy of nanostructural materials with negative curvature and related size effects. Chemical reviews 2009, 109 (9), 4221-4247.

55. Wulff, G., On the question of speed of growth and dissolution of crystal surfaces. Z. Kristallogr 1901, $34(5 / 6), 449$.

56. Kim, J.; Manna, S.; Dietze, S.; Ulvestad, A.; Harder, R.; Fohtung, E.; Fullerton, E.; Shpyrko, O., Curvature-induced and thermal strain in polyhedral gold nanocrystals. Applied Physics Letters 2014, 105 (17), 173108.

57. Baber, A. E.; Jensen, S. C.; Iski, E. V.; Sykes, E. C. H., Extraordinary atomic mobility of $A u\{111\}$ at 80 Kelvin: effect of styrene adsorption. Journal of the American Chemical Society 2006, 128 (48), 15384-15385. 58. Zhao, L.; Ji, X.; Sun, X.; Li, J.; Yang, W.; Peng, X., Formation and stability of gold nanoflowers by the seeding approach: the effect of intraparticle ripening. The Journal of Physical Chemistry C 2009, 113 (38), 16645-16651.

59. Wu, H.-Y.; Liu, M.; Huang, M. H., Direct synthesis of branched gold nanocrystals and their transformation into spherical nanoparticles. The Journal of Physical Chemistry B 2006, 110 (39), 1929119294.

60. Xiong, G.; Clark, J. N.; Nicklin, C.; Rawle, J.; Robinson, I. K., Atomic diffusion within individual gold nanocrystal. Scientific reports 2014, 4, 6765.

61. Marks, L.; Peng, L., Nanoparticle shape, thermodynamics and kinetics. Journal of Physics: Condensed Matter 2016, 28 (5), 053001. 